\title{
Avoiding a crunch
}

\author{
Fears of an imminent capacity crunch in optical networks may be alarmist but new more efficient \\ technologies for transporting data will be required in the future.
}

The introduction of dense wavelength division multiplexing in the $1.5 \mu \mathrm{m}$ band in the $1990 \mathrm{~s}$ opened up transmission capacity on the scale of terabits per second for optical fibre communication systems. Although considered by many to be an effectively unlimited resource at the time, it is now widely accepted that this is no longer the case. A common and frequently heard concern is that we will soon exhaust the bandwidth associated with a standard single-mode optical fibre - a worry being fuelled by a seemingly endless rise in the use of the Internet, e-mail and other data-rich applications, especially with mobile devices.

"The number of Internet users is growing linearly and has now reached around three billion - half of the world's population," Fatima Garcia Gunning from University College Cork told attendees at The Royal Society's Communication Networks Beyond the Capacity Crunch meeting, which took place during 11-14 May (http://go.nature.com/4eKIYy). "The entire network needs to become more energy efficient."

Although doomsday-fears that a capacity crunch is imminent are undoubtedly exaggerated and unlikely to come to pass (see the News \& Views on page 424), in the long term new technological solutions are going to be required to allow transmission on the scale of petabits per second but perhaps even more importantly drive down the cost and energy of transporting a bit of data from A to B. The bottom line is that we need to become more efficient and flexible with how we transport and switch data around networks.

"Optical data traffic will increase by orders of magnitude in the future, and there is an urgent need to increase transmission capacity while decreasing cost and energy consumption per bit," commented Joseph Kahn from Stanford University, an optical communications expert who wasn't at the meeting but spoke with Nature Photonics independently. "In the end, I believe that the cost (and perhaps energy) per bit may not drop as fast as the traffic increases, and we may need to figure out how to pay the higher aggregate cost."

As a result, it should come as no surprise that the photonics community is now seriously considering what strategies can be employed, and in particular what new hardware will be required, in the future.

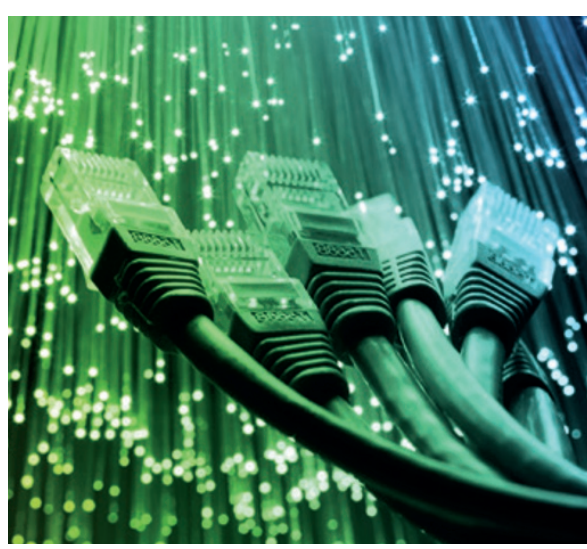

Fortunately, Kahn believes that we do have at our disposal the technologies and ideas that are needed to help scale capacity and avoid a crunch. "The capacity increase will be achieved by using more fibres and judiciously increasing the capacity per fibre. The capacity per fibre can be increased by using more bandwidth for wavelength division multiplexing, by increasing the signal-to-noise ratio and by using higherorder modulation to increase spectral efficiency, or by exploiting multiple cores or multiple spatial modes in a single fibre, that is, spatial multiplexing. Reductions in cost and energy consumption will be achieved especially through component integration."

Indeed, the principles and current status of many of these approaches were heavily discussed at The Royal Society meeting.

The two most discussed approaches for scaling capacity are: (i) broadening the telecoms spectral transmission window beyond the $1.5 \mu \mathrm{m}$ region currently supported by standard optical fibre and erbium-doped fibre amplifiers; (ii) increasing the number of spatial transmission channels through the use of fibres with multiple cores or with fibres that support multiple modes, or even both. The former requires the development of a lowloss fibre design and optical amplifiers that operate at new wavelengths.

Fortunately, considerable progress has taken place on both of these fronts and prototype hollow-core photonic bandgap fibres, which offer a much broader transmission of hundreds of nanometres, have already been developed. The best of these still have a loss about ten times larger than standard fibres and have only been drawn in lengths up to a few kilometres, but the progress is encouraging. Fibres doped with thulium or bismuth have also shown promise for amplifying signals either side of erbium's $1.5 \mu \mathrm{m}$ window at around $2 \mu \mathrm{m}$ and $1.2 \mu \mathrm{m}$, respectively.

"Keep your eye on the combination of bismuth and rare-earth ions [erbium or thulium] as this should make it possible to cover the entire $1-2 \mu \mathrm{m}$ region," commented David Richardson from the University of Southampton. "A continuous gain from the $\mathrm{C}$ band [1.52-1.56 $\mu \mathrm{m}$ ] to $2.1 \mu \mathrm{m}$ should be achievable within the next two to three years."

One open question is whether unlocking a wider spectral transmission window will prove to be economically viable and attractive to telecom operators. "Exploitation of new wavelength bands can certainly increase transmission capacity per fibre, but it is not obvious that this alone will address all the challenges. We have had both the $\mathrm{C}$ and $\mathrm{L}[1.57-1.61 \mu \mathrm{m}]$ bands for many years, but most telecom carriers (in the USA, at least) prefer to use only the $\mathrm{C}$ band, lighting up new fibres as needed," commented Kahn.

The other approach of spatial multiplexing has also attracted great interest in recent years, with a large European project (MODEGAP) on the topic and several large telecom firms exploring the idea (Alcatel-Lucent's Bell Labs, NTT and Sumitomo, among others). The benefits in terms of an increase in transmission capacity are potentially very large but the challenges associated with multiplexing and demultiplexing a large number of modes and dealing with modal dependent loss and crosstalk are not trivial to overcome. "Spatial multiplexing was oversold in some ways. For example, it was stated that because the capacity per single-mode fibre in long-haul systems is approaching limits, we must increase the capacity per fibre by spatial multiplexing," commented Kahn. "Nevertheless, spatial multiplexing offers compelling advantages, and may well be used widely. The most obvious near-term application is in data centres or computer backplanes, where multicore fibres may help increase interconnection density. In submarine systems, limitations on the cable diameter may favour multicore fibres or mode-division multiplexing." 